\title{
EXPLORING PLAY INTERVENTIONS IN DESIGN EDUCATION
}

\author{
Garrett HAZEN, David MORGAN and Bryan HOWELL \\ Brigham Young University
}

\begin{abstract}
This study reports a collection of surveyed responses from industrial design students at Brigham Young University over six semesters regarding high levels of perceived stress in a competitive class environment, and it outlines playful interventions in coursework and classroom settings to measure if play can make any significant impact on students' perceptions of stress and feelings of frustration. This study is inspired by existing play studies that continue to demonstrate the positive relationship between the freedom to be playful and psychological wellbeing. The methods used in this study include a consolidation of 99 anonymous free-response student surveys over the course of six semesters that are organised in terms of stress level and frustration word indicators. These comments provide a historical baseline for understanding the student perceptions of their classroom learning experience as it relates to their emotional wellbeing. We then compare those comments with comments made after a semester of playful interventions to discover how they differ. The results indicate that levels of "stress" and "frustration" were decreased with playful interventions, however students spent more hours outside of class lecture on their assignments and the overall student course rating nominally decreased. These results are discussed as well as how pedagogical changes to traditional classroom environments such as this may not only impact stress and frustration but also significantly contribute to the holistic learning experience for university students.
\end{abstract}

Keywords: Industrial design, student learning, studio environment, creativity pedagogy, class room design

\section{INTRODUCTION}

The course discussed in this study is one of four courses that are designed to educate freshman industrial design students on the basic principles of design and provide them the materials and opportunities to develop a competitive portfolio for admission into the professional programme. The nature of these courses is highly competitive, admitting only 15-18 students per year out of 25-35 applicants. Using six semesters worth of university sponsored course ratings reports submitted by students we have evidence indicating that these courses create an environment that increases levels of student stress and frustration and a substantial number of hours spent outside of class lecture, even though the course is highly rated. Concerned by the contrasting affects these courses appear to be having on students, we are exploring ways to improve the curriculum and classroom environments to decrease stress and frustration, while providing students an environment that maximises their learning experience.

We chose to address these issues through play. Surveys have shown students from the Industrial Design programme at Brigham Young University (BYU) innately have a higher playful disposition than students who study in our neighbouring engineering programme. Because of this, we felt it logical to select one of the core courses and make playful interventions into the classroom environment and learning exercises to see if we can decrease stress and frustration levels among students in the current course compared with students from years past.

Play studies demonstrate the positive relationship between the freedom to be playful and psychological wellbeing [1]. Because recent studies suggest academic environments can have negative impacts on wellbeing [2], designing playful interventions into academic settings may positively impact college level students' levels of perceived stress. Playful behaviour is characterised by physical, psychological, and emotional attitudes toward things, people, and situations that are less serious but still respect the purposes and goals of the objects or contexts [3]. Researchers are beginning to recognise and measure 
the benefits of play in stressful environments, their findings suggesting that "those who play will gain important benefits for their capability for innovation and better adaptivity at the face of quick and surprising changes," [4]. Recent research measuring playfulness in academic environments has found that adult playfulness contributes positively to academic performance [5] and that adult individuals who exhibit lower levels of playfulness experience higher levels of perceived stress than individuals with medium or high levels of playfulness [6]. Further, "playful individuals more frequently utilised adaptive, stressor focused coping strategies and were less likely to employ negative, avoidant, and escape oriented strategies" [6].

We hypothesise that students who participate in the playful interventions will report a decreased level of stress and frustration in the open response section in the end of semester course ratings. We further believe that these interventions could encourage discussions amongst educators to incorporate playful interventions into their courses.

This paper outlines the methods used to obtain and measure student levels of stress, frustration and playfulness and categorise cohorts of students as more or less playful. The paper will discuss their meaning and how this study contributes to a larger body of current research that aims to provide educators the tools to make pedagogical improvements in their teaching. Modern scholars no longer emphasise knowledge acquisition and memorisation as sacrosanct, arguing that a technology and information based society requires an education that emphasises "development of critical analysis competencies, organising facts by communicating and cooperating with other professionals, and discovering new information to create novel ideas" [7]. As education adapts to changes in culture and society, educators should also adapt and develop new methods to engage students in impactful ways. This study on playful interventions in an academic setting is intended to fit into the growing body of research that supports a shift in educational practices that can positively impact student learning.

\section{METHODS}

\subsection{The Short Measure of Adult Playfulness (SMAP)}

The Short Measure of Adult Playfulness (SMAP) is a five item questionnaire designed to assess playfulness in adults through understanding playful experiences and a display of playful activities. All items (see Table 1) are positively keyed and utilise a four point scale ( 1 = "strongly disagree," 4 = "strongly agree"). Item 1: "I am a playful person"; 2 : "Good friends would describe me as a playful person"; 3: "I frequently do playful things in my daily life"; 4: "It does not take much for me to change from a serious to a playful frame of mind"; 5: "Sometimes, I completely forget about the time and am absorbed in a playful activity." Details about the content and analysis of the SMAP can be found in Proyer's paper from 2011 [5]. We administered the questionnaire to 20 undergraduate industrial design students and 15 undergraduate and 5 graduate mechanical engineering students. Our study reports a higher playfulness score in design students than in engineering students as shown in Table 1. This finding indicates that incorporating playful interventions into a design course could be a promising way to contribute to the holistic learning experience for participating design students.

Table 1. Short Measure of Adult Playfulness (SMAP)

\begin{tabular}{|c|c|c|c|c|}
\hline & $\begin{array}{c}\text { Sample 1 } \\
\text { (Proyer) }\end{array}$ & $\begin{array}{c}\text { Sample 2 } \\
\text { (Proyer) }\end{array}$ & $\begin{array}{c}\text { Mechanical Engineering } \\
\text { Students }\end{array}$ & $\begin{array}{c}\text { Industrial Design Students Before } \\
\text { Interventions }\end{array}$ \\
\hline SMAP Mean & 2.77 & 3.03 & 2.88 & 3.24 \\
\hline SD & .64 & .89 & .65 & .71 \\
\hline
\end{tabular}

\subsection{University Course Ratings}

Students are asked by BYU to complete a course ratings survey at the end of each semester for classes they attended. This survey is standardised for all university courses and covers course and instructor performance assessments. The last section of the survey allows students to provide free responses to anything the students wish to comment on.

For this study we gathered the course ratings reports from Fall 2015 through Winter 2018 or the last 6 semesters for this particular course. Of the 119 students who participated in the course, 99 of them completed the survey. While these surveys do not specifically cover "emotional outcomes" or the notion 
of "play," we felt that by searching the free response sections completed by the students we could identify and tally words that would reflect the student's emotional and play reactions to the course and would then compare the average use of these identified words from the past 6 semesters to their use as recorded from the semester in which the play interventions were inserted.

Using the survey results, we identified two notions that reflected emotional outcomes from the class that we felt could be altered by introducing play into the course: personal perceptions of stress and expressions of frustration with the course or coursework. These notions were either called out specifically by the words "stress" and "frustration" or alluded to through a combination of closely associated words. From the surveys, which had an extraordinary response rate of $83 \%$, we found 46 references of 'stress', an average of 7.7 students per class and 12 references of 'frustration' an average of 2 students per class.

The standardised section of the survey also provides an overall composite rating for the course with 5 being the highest possible score as well as the number of hours students report they spent on work outside of class. The six semester average course rating for this course has a 4.35 course rating and students report they spent more than 10 hours outside of class lecture. We feel assessing these two indicators before and after introducing "play" into the curriculum might also indirectly demonstrate how "play" affected the course outcomes.

\subsection{Course Playfulness Interventions}

Playful interventions embrace an approach to teaching and learning that "stimulates intrinsic motivation and educational drive, creates safe spaces for academic experimentation and exploration, and promotes reflective risk taking, ideation, and participation in education" [8]. Incorporating these interventions into established course assignments and practices encourage students to engage in active learning in enjoyable ways.

\subsubsection{Class Practice and Studio Space Intervention}

For this study we created and introduced interventions of play into the Introduction to 3D Design course by manipulating class practices, coursework and the physical environment.

On the first day of class we set a precedent that this course should be perceived as playful by playing a "surprise me" game, which involves each student in turn telling the others something about themselves that may be surprising. This activity is intended as an amusing "get-to-know-you" activity that is part of establishing a safe space for student exploration to occur.

We also initiated a classroom group project where students collaboratively built a wooden geodesic dome structure in order to create a space somewhat apart from the larger studio, which will remain in place until the end of the course. This dome offers a location for relaxed, self-determined activity, and implies ownership by the students who built it. They were encouraged to actively modify the dome to fit their creative desires and participate in the creation of their learning space. See Figure 1.
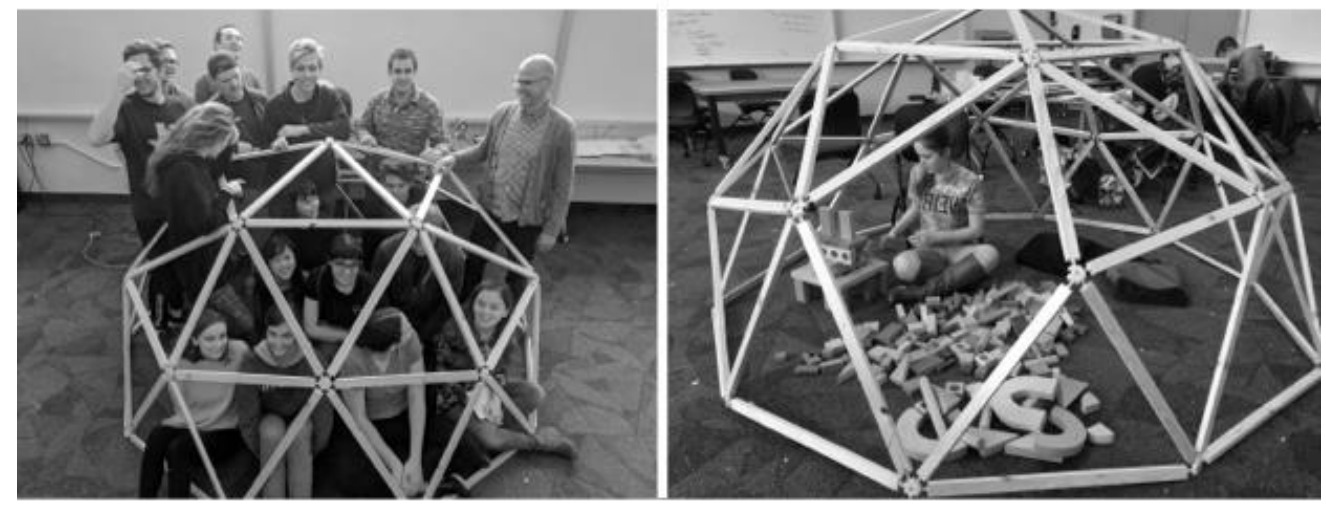

Figure 1. Students building and using the geodesic dome in the studio (left), and students playing with blocks beneath the geodesic dome (right)

Finally, we instituted an additional assessment method at the end of each assignment. In addition to class critique we exhibited each student's project in an informal gallery display, which rotates with each assignment throughout the course. The gallery provides an additional context in which the students efforts can be evaluated. It also gives beginning students a chance to present their work to a larger audience. The written comments from gallery visitors has provided a lively channel of public feedback 
encouraging participation in the larger dialogue of their design education as shown on the left image of Figure 2.
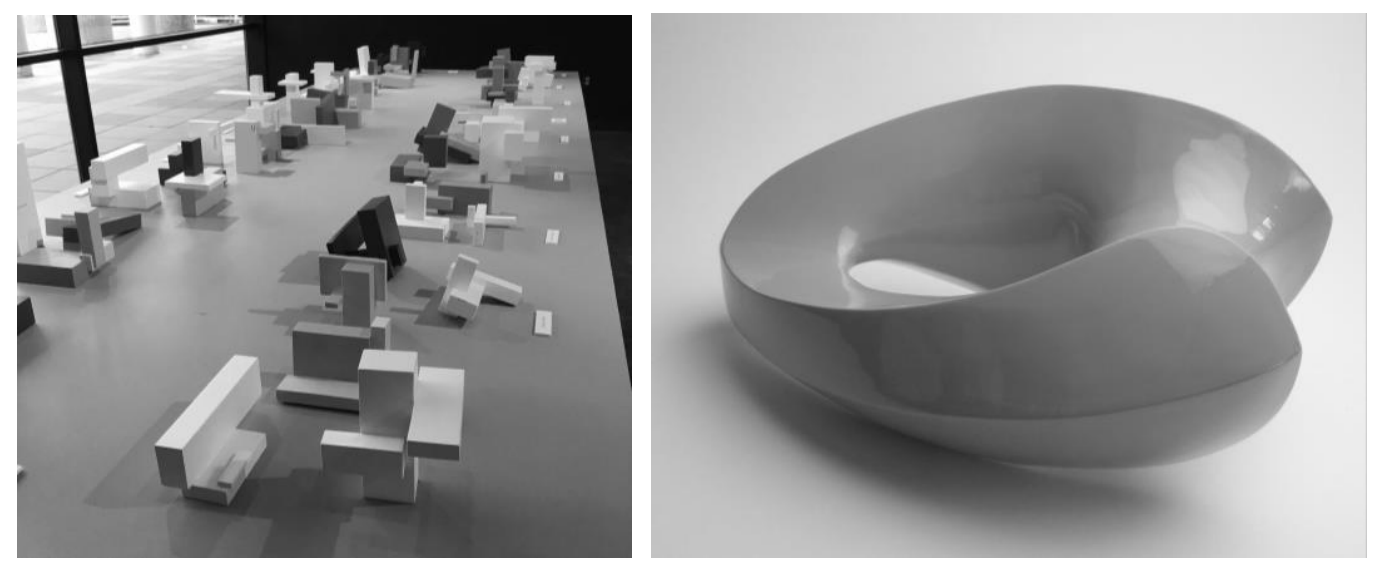

Figure 2. Informal gallery and display of student work on the block assignment (left), and an example of a student's final abstract "bone form" submission (right)

\subsubsection{Playful Changes to Coursework}

Coursework and assignments over the past six terms in the course has remained consistent, with minimal adjustments made occasionally due to timing or other considerations. During the eight-week course, we kept the same assignments but adjusted the way they were presented to the class in order to provide students a more flexible and playful means through which they could accomplish them.

An early assignment is to make a folded mask from paper. This assignment explores how twodimensional patterns become three-dimensional objects, and how those resultant objects need to respond to human morphology. Usually the assignment assumes a symmetrical design, but in an attempt to encourage risk taking, our playful intervention was to introduce the possibility of asymmetry into the mask design, giving students more freedom to invent and play as they saw fit.

Another assignment is to create a composition using rectilinear rigid foam blocks that intersect threedimensionally in a visually dynamic way. Rather than asking the students to start with the foam material, we had them begin by building compositions using wooden blocks. The goal was to provide a more immediate and accessible path to generating multiple quick compositional ideas, without the need to use tools and develop technique immediately. This approach was intended to progressively connect the fun of playing with blocks to the more rigorous process of compositional iteration.

We wondered if perhaps the playful activity could lead to greater idea fluency and consequently better quality compositions as shown in the right image of Figure 1.

A concluding assignment for the course is to create an abstract sculptural object based on the form language of mammal bones. Typically, students search for and collect intact bone remains of local wildlife to study their aesthetic and structural properties. For this study we incorporated a new group aspect of the assignment where students created made up speculative skeletons of imagined creatures using the collected bones. This addition encouraged further experimental and explorative engagement with the physical artefacts in an active and enjoyable way.

\section{RESULTS}

Table 2. Comparison of indicators between previous courses and current course

\begin{tabular}{|c|c|c|c|c|}
\hline & Stress & Frustration & Course Rating & $\begin{array}{c}\text { Hours Spent Outside } \\
\text { of Class Lecture }\end{array}$ \\
\hline $\begin{array}{c}\text { Average ratings of } \\
\text { previous six courses }\end{array}$ & 7.7 & 3.5 & 4.35 & 10.25 \\
\hline $\begin{array}{c}\text { Average ratings of } \\
\text { current course }\end{array}$ & 5 & 2 & 4.0 & 11.90 \\
\hline Difference & $\mathbf{- 2 . 7}$ & $\mathbf{- 1 . 5}$ & $\mathbf{- . 3 5}$ & $\mathbf{+ 1 . 6 5}$ \\
\hline
\end{tabular}


According to students' free responses to university administered surveys, there was a positive decrease in both notions of "stress" and "frustration" among students who participated in the course with playful interventions. However, the average overall course rating slightly decreased, and students spent more time on their projects outside of classroom lecture time than students in previous versions of the course. Whether this increase in hours spent is positive or negative merits further discussion.

\section{DISCUSSION}

This study relies on free response surveys and the numbers shown do not reflect the full nuance of a student's individual experience. That said, the results show that reported levels of "stress" and "frustration" in the course were reduced, thus potentially impacting learning. The playful interventions discussed may have had some beneficial effect on these measurements, but students did not mention these interventions directly in the university administered surveys, commenting instead that the projects were "super fun" and that "of the four classes, I probably enjoyed this one the most." While students spoke less of their stress and frustration, they did express confusion about what was expected of them. More ambiguity may have been introduced because the playful interventions loosened the structure of the assignments. Additionally, our study lacks insight on how playful interventions may have helped students analyse their final project results or how to improve their designs in the future. Several students commented, however, that they felt they "learned a lot" and that the course required them to "use a lot of brain power." Increased ambiguity as a result of the interventions may have invited students to adapt and think critically about their design decisions. Further study on the relationship between ambiguity and play in the university classroom may reveal more insight on how play can impact student learning. The slight decrease in course rating may be attributed to several possible factors, such as poorer performance by the professor, coursework ambiguity, or a possible decrease in overall effectiveness brought about by introducing "play" methods. Given the relatively small change in the rating, it is more likely due to variance in the personalities of the participating cohort. The increase in hours spent outside of class may have been influenced by the interventions. Students in these courses already spend an arguably unbalanced amount of time on class assignments outside of lecture, so the increase could be seen as an unfavourable addition of workload for students who may already be overworked. However, considering that stress levels went down, it is possible that the workload stayed the same, but the interventions invited students to spend more time in the explorative phases of their design. One student stated that they "enjoyed the work and learned how to enjoy the creative process more."

Building the geodesic dome provided an enjoyable activity at the beginning of class. Students were enthusiastic participants and immediately wanted to spend time in the space. The dome was somewhat less used for assignments as the course progressed, although it continually provided an alternative space used for relaxing and non-design activity and added a quirky presence in the studio. Similarly, it does not appear that the addition of wooden blocks had any noticeable effect on idea generation or the quality of the final block compositions, but students continued to play with them freely as the course progressed. The inviting and playful presence of both dome and blocks may have provided a relaxing element to the space, which in turn potentially impacted how students perceived their sense of stress and frustration throughout the course.

Exhibiting all student work in the informal gallery proved to be a major new element of the course. We observed that while a few students felt indifferent about having their work on display, most were excited to see their projects presented to the university community in this way. It appears the practice of setting up each show (five in total) provided many students a light-hearted outlet for expression at the end of each project, perhaps as a positive way to frame their work after a demanding final class critique.

Some playful intervention attempts were not successful. Although we mentioned to students the possibility of exploring asymmetry in their mask designs, not one student produced an asymmetrical design. We are not sure why, but we felt it was important not to push them to accept the interventions we planned. Our interest was to propose a range of playful interventions and observe which ones contributed to a more playful environment. Our attempt to encourage students to make up speculative skeletons with the found bones they collected failed completely. One particular student was very vocal against the idea, announcing to us all how "disgusting" it was to handle the bones any more than necessary. The other students seemed somewhat surprised by her strong opinion but did not offer their own. No made up skeletons were created.

The playful interventions described in this paper were implemented in a course that emphasises form generation and surface analysis. It is natural to include a more flexible structure to coursework within a 
programme that emphasises these skills, and it may feel less natural in an engineering field where environments may be more austere and highly structured coursework is required.

The study of play activities and playfulness has been primarily associated with children and only recently has been more seriously studied in its relationship to adults [8]. Emerging studies are beginning to indicate that understanding pre-school playfulness has a lot to offer educators in higher education, such as how to create environments that encourage students to fail quickly, make discoveries, and solve problems through playful means. Changes to teaching methods that encourage this type of behaviour in students are emerging. For example, the University of Portsmouth "uses 'pervasive learning' activities, where courses are taught through playful, detailed simulations in which students work together to solve problems and make mistakes away from the real consequences of assessment" [9].

Educators have the ability and freedom to manipulate coursework and the classroom environment to benefit student learning and engagement. Testing student abilities to recall information is becoming less relevant in a world where information is easy to locate. A more difficult and relevant task is teaching students to competently adapt to and navigate through new contexts and information. Therefore, we hope that the methods described in this paper can provide insight and inspiration for educators who wish to benefit the holistic student learning experience through new ways of teaching.

\section{CONCLUSIONS}

Playfulness is a human characteristic that remains relevant for learning beyond childhood. As the results of this study appear consistent with the results of other emerging studies on the potential benefits of play in adults, college level educators who seek to adapt their teaching methods to create an engaging and healthy atmosphere for their students could benefit from the insights provided here and may be encouraged to explore playful interventions of their own.

\section{REFERENCES:}

[1] Glynn M. and Webster J. The adult playfulness scale: An initial assessment. Psychological Reports, 1992, 71, 83-103.

[2] Icaro J.S.R., Pereira I.V.F., de Oliveira B.G., Casotti C.A. and Boery E.N. Stress and Quality of Life Among University Students: A Systematic Literature Review. Health Professionals Education, 2017, 4(2), 70-77.

[3] Sicart M. Play Matters, 2014, (The MIT Press, Cambridge, Massachusetts).

[4] Nummenmaa T., Savolainen S. and Kultima A. Towards Playful Office Culture, Final Report of the OASIS - Playful Spaces for Learning and Collaboration at Work (2014-2015) research project, 2015, (University of Tampere, TRIM).

[5] Proyer R.T. Development and Initial Assessment of a Short Measure for Adult Playfulness: The SMAP. Personality and Individual Differences, 2012, 53(8), pp. 989-994.

[6] Magnuson C.D. and Barnett L.A. The Playful Advantage: How Playfulness Enhances Coping with Stress, Leisure Sciences, An Interdisciplinary Journal, 2012, 35(2), 129-144

[7] Park E.L. and Choi B. K. Transformation of classroom spaces: Traditional versus active learning classroom in college, Higher Education, 2014, 68, 749-771.

[8] Nørgård R.T., Toft-Nielsen C. and Whitton N. Playful learning in higher education: developing a signature pedagogy, International Journal of Play, 2017, 6(3), 272-282.

[9] Whitton N. The Importance of Play: What Universities Can Learn from Pre-School. Available: https://www.theedadvocate.org/importance-play-universities-can-learn-preschools/ [Accessed on 2018, 11, March]. 